\title{
DISPONIBILIDADE DE CÁTIONS NO SOLO ALTERADA PELO SISTEMA DE MANEJO ${ }^{(1)}$
}

\author{
Paulo Sergio Pavinato ${ }^{(2)}$, Alexandre Merlin ${ }^{(3)}$ \& Ciro Antonio Rosolem ${ }^{(4)}$
}

\begin{abstract}
RESUMO
O sistema de manejo normalmente afeta a disponibilidade de nutrientes no solo, em decorrência das práticas de cultivo, da rotação de culturas e dos resíduos remanescentes na área cultivada. $O$ objetivo deste trabalho foi avaliar as alterações na disponibilidade de cátions no perfil do solo sob sistema convencional e semeadura direta, por mais de 10 anos, em dois Latossolos do cerrado brasileiro. Os experimentos foram realizados em Costa Rica, MS, e Luziânia, GO, sendo um Latossolo Vermelho (LV) e um Latossolo Vermelho-Amarelo (LVA), respectivamente, com dois sistemas de cultivo, semeadura direta (SSD) e cultivo convencional (SCC). Os solos foram coletados em tubos de PVC, da superfície até $40 \mathrm{~cm}$ de profundidade, e conduzidos para casa de vegetação, no Departamento de Produção Vegetal, FCA-UNESP, Botucatu-SP, sendo cultivada a soja sobre esses vasos por 60 dias, com e sem aplicação de fosfato, totalizando oito tratamentos, com quatro repetições. As avaliações foram feitas por meio de coletas da solução do solo durante o período de cultivo da soja e análises de solo após o cultivo. Na solução do solo foram avaliadas as concentrações de $\mathrm{K}$, Ca e Mg. Nas amostras de solo, coletadas nas profundidades de 0-5, 5-10, 10-15, 15-25 e 25-40 cm, foram determinados o $\mathrm{pH}, \mathrm{MO}, \mathrm{K}, \mathrm{Ca}^{2+}, \mathrm{Mg}^{2+} \mathrm{e}^{3+}$. O sistema de cultivo promove mudanças na disponibilidade de $K$ na camada considerada arável do perfil do solo, sendo observada menor disponibilidade no sistema de semeadura direta em relação ao sistema convencional. Solos com camadas superficiais mais argilosas, caso do LV, tem potencial de manter maior teor de $\mathrm{K}$ disponível nestas camadas. Nos solos estudados, a disponibilidade de Ca e Mg é maior na camada superficial (0-5 cm) no sistema plantio direto, em consequência da disponibilização desses nutrientes dos resíduos em decomposição. Para o sistema convencional, a disponibilidade é menor na superfície, mas ainda em teores considerados altos, e é mantida em toda a camada arável do perfil.
\end{abstract}

Termos de indexação: acidez e manejo do solo, potássio no solo, cálcio no solo, magnésio no solo, semeadura direta.

\footnotetext{
(1) Trabalho extraído da tese de doutorado do primeiro autor, apresentada à Universidade Estadual Paulista - UNESP. Projeto financiado pela FAPESP. Recebido para publicação em outubro de 2008 e aprovado em maio de 2009.

(2) Professor da Universidade Tecnológica Federal do Paraná - UTFPR. Estrada para Boa Esperança, km 04, CEP 85660-000 Dois Vizinhos (PR). E-mail: pavinato@utfpr.edu.br

(3) Pós-Graduando no Departamento de Produção Vegetal, Faculdade de Ciências Agronômicas. CEP 18603-970 Botucatu (SP). E-mail: amerlin@fca.unesp.br

(4) Professor do Departamento de Produção Vegetal, Faculdade de Ciências Agronômicas. E-mail: rosolem@fca.unesp.br
} 


\title{
SUMMARY: SOIL CATIONAVAILABILITYAS AFFECTED BY THE TILLAGE SYSTEM
}

\begin{abstract}
The management system normally affects soil nutrient availability, as a consequence of tillage practices, crop rotation and crop residues remaining in the cultivated area. The objective of this study was to evaluate the alterations in cation availability in two Oxisols in the Brazilian Cerrado under conventional and no-tillage systems for more than 10 years. The experiments were conducted in Costa Rica, state of Mato Grosso do Sul, and in Luziânia, Goiás. Both soils were Oxisols, but one being a Red Latosol (LV) and the other a Red-Yellow Latosol (LVA), respectively, under two management systems, no-tillage (SSD) and conventional (CV). Soils were collected in PVC tubes from the surface to a depth of $40 \mathrm{~cm}$, and taken to a greenhouse of the Departamento de Produção Vegetal, FCA-UNESP, Botucatu-SP. In these tubes, soybean was grown for 60 days, with and without phosphate application, totaling eight treatments ( $2 \times 2 \times 2)$, with 4 replications. The evaluations were based on the soil solutions collected during soybean growth to evaluate $K, C a$ and $M g$ concentrations and on soil analysis after crop harvest (sampled from the layers $0-5,5-10,10-15,15-25$ and $25-40 \mathrm{~cm}$ ), to determine $\mathrm{pH}$, $\mathrm{OM}, \mathrm{K}, \mathrm{Ca}^{2+}, \mathrm{Mg}^{2+}$, and $\mathrm{Al}^{3+}$. The tillage system led to changes in Kavailability in the arable depth layer, where lower availability was observed in no-tillage than in conventional systems. In soils with clayey surface layers, such as the LV soil, the potential to maintain higher available Klevels in these layers is greater. In the studied soils the Ca and Mg availability is higher in the upper layers $(0-5 \mathrm{~cm})$ under no-till as a consequence of the availability of these nutrients from residue decomposition. In the conventional system the availability is lower in the surface layer, although still high, and remains stable in the entire arable soil depth.
\end{abstract}

Index terms: acidity and soil management, soil potassium, soil calcium, soil magnesium, notillage.

\section{INTRODUÇÃO}

A disponibilidade de nutrientes no solo é normalmente afetada pelas alterações sofridas pelo solo em decorrência das práticas de cultivo e das rotações de culturas adotadas pelos produtores. Nos últimos anos, tem aumentado a área cultivada sob sistema de semeadura direta no Brasil, por ser um sistema mais sustentável com relação à conservação do solo e ciclagem de nutrientes pelas culturas.

A adoção de práticas conservacionistas normalmente tem efeito positivo sobre o teor e a qualidade da matéria orgânica do solo (Bayer \& Mielniczuk, 1997), com reflexos diretos ou indiretos sobre as características químicas (Frazão et al., 2008). Em áreas com uso do sistema plantio direto, preconizase a manutenção de resíduos culturais sobre o solo e a mínima alteração da sua estrutura, para minimizar seu contato com o solo e diminuir, com isso, a velocidade de decomposição desses resíduos. A adoção dessas práticas em solos arenosos e, ou, argilosos com pouco aporte de matéria vegetal durante o ano, como é o caso das regiões do cerrado brasileiro, principalmente em regiões de inverno seco, pode aumentar ou pelo menos manter sua capacidade produtiva, em razão do possível aumento na CTC do solo e da maior disponibilidade de nutrientes às plantas em função do acúmulo de MOS (Bayer \& Mielniczuk, 1997; Frazão et al., 2008).

No sistema de semeadura direta, o suprimento de nutrientes pela decomposição dos resíduos e também a interrupção do ciclo de doenças e o controle de ervas têm sido apresentados como algumas das vantagens pela rotação de culturas e pelo uso de culturas de cobertura no inverno. Além disso, tem sido observado que os resíduos de cobertura favorecem a mobilização de cátions e sua disponibilização para as culturas posteriores (Franchini et al., 2003).

A acidez do solo é, na maioria das vezes, um dos principais limitantes da produção em áreas agrícolas brasileiras. A deposição de resíduos vegetais pode promover a elevação do $\mathrm{pH}$ do solo na camada superficial, pela troca ou complexação dos íons $\mathrm{H}$ e $\mathrm{Al}$, por $\mathrm{Ca}, \mathrm{Mg}, \mathrm{K}$ e outros compostos presentes no resíduo vegetal, aumentando assim a saturação por bases (Amaral et al., 2004). As reações de troca de ligantes entre ânions orgânicos e os grupos funcionais dos óxidos de $\mathrm{Fe}$ e $\mathrm{Al}$ têm sido propostas como uma das causas da elevação de $\mathrm{pH}$ do solo após a adição dos resíduos de plantas (Franchini et al., 1999).

Com isso, a formação e manutenção da palhada pelo sistema de semeadura direta podem favorecer a disponibilidade de nutrientes no solo. Kretzschmar et al. (1991) demonstraram que a mistura de palha de milheto com o solo aumentou o $\mathrm{pH}$ da solução e reduziu a disponibilidade de $\mathrm{Al}^{3+}$. Recentemente, outros resultados confirmaram isso, pois a adição de resíduos vegetais aumentou os valores de $\mathrm{pH}$ e teores de $\mathrm{Ca}^{2+}, \mathrm{K}^{+}, \mathrm{Mg}^{2+}$, além de reduzir os teores de $\mathrm{Al}$ trocável no solo (Franchini et al., 1999). Entretanto, estes efeitos parecem ocorrer em curto prazo, como mencionado por Miyazawa et al. (1993), os quais 
observaram que o material vegetal causou aumentos rápidos no $\mathrm{pH}$ imediatamente após a adição do resíduo, havendo a diminuição rápida e gradativa até a estabilização, quando resíduos de várias espécies de plantas foram adicionados ao solo. Desta forma, há evidências de que os resíduos vegetais podem atuar diretamente na redução da acidez do solo no aumento de $\mathrm{pH}$, na mobilidade de cátions básicos ou na complexação de $\mathrm{Al}$. Com isso, o objetivo deste trabalho foi avaliar alterações na disponibilidade de cátions no perfil do solo sob sistema de cultivo convencional e de semeadura direta, por mais de 10 anos, em dois Latossolos do cerrado brasileiro.

\section{MATERIAL E MÉTODOS}

Os experimentos de campo foram realizados em Costa Rica, MS (latitude $18^{\circ} 12^{\prime} 52$ ” S, longitude $53^{\circ} 11^{\prime} 50$ " O e altitude média de 859 m) e Luziânia, GO (latitude $16^{\circ} 13^{\prime} 43^{\prime} \mathrm{S}$, longitude $47^{\circ} 37^{\prime} 28$ " $\mathrm{O}$ e altitude média de $988 \mathrm{~m}$ ), sendo os solos um Latossolo Vermelho (LV) e um Latossolo VermelhoAmarelo (LVA), respectivamente (Embrapa, 1999). Em cada local, foram coletadas, em janeiro de 2005 , amostras representativas de dois sistemas de manejo do solo, semeadura direta (SSD) e cultivo convencional (SCC), ambos há pelo menos 10 anos sob cada sistema de cultivo e sob as rotações de culturas descritas a seguir.

O solo LVA vinha sendo cultivado, desde o ano agrícola 92/93, nos sistemas de semeadura direta e de cultivo convencional (aração com arado de disco até $20 \mathrm{~cm}$, com posterior uso de duas gradagens niveladoras), com a sucessão de soja e milho como culturas principais de verão e inverno, com pouca cobertura, apenas de vegetação espontânea, pois a região é extremamente deficiente em precipitação pluvial no período de abril a setembro. No caso do solo LV, a área de coleta estava sob cultivo sucessivo de milho-algodão-soja no verão, com algumas culturas de inverno no período, como o nabo forrageiro antecedendo o milho ou algodão também nos sistemas direto e convencional (com mesmos implementos e práticas usados no solo LVA), desde 1994.

Os solos foram coletados em cada local descrito anteriormente, com a penetração de tubos de PVC de $20 \mathrm{~cm}$ de diâmetro e $42 \mathrm{~cm}$ de comprimento, sendo inserido até $40 \mathrm{~cm}$ de profundidade, com posterior extração destes tubos com solo, mantendo praticamente intacta a estrutura do solo internamente ao tubo. No momento da coleta das unidades experimentais, foram tomadas amostras de ambos os solos nas profundidades de $0-5,5-10,10-15,15-25$ e 25-40 cm, cuja fertilidade (Raij et al., 2001) e granulometria (Embrapa, 1997) foram caracterizadas (Quadro 1).
A soja foi cultivada nas amostras de solos nesses tubos, pelo período de 60 dias, em casa de vegetação localizada no Departamento de Produção Vegetal, FCAUNESP, Botucatu-SP, objetivando avaliar a disponibilidade de $\mathrm{P}$ com o efeito do manejo adotado por longo tempo nesses solos, objeto principal de outro estudo (Pavinato et al., 2008). Portanto, assume-se que os tratamentos com $\mathrm{P}$ aplicados em casa de vegetação praticamente não alteraram a disponibilidade dos cátions e a acidez do solo, originais do campo onde se coletaram as amostras, por ser por período relativamente curto e por não haver adição de fertilizantes com os nutrientes avaliados neste trabalho, com exceção do Ca do superfosfato simples. Os tratamentos aplicados em casa de vegetação foram assim compostos: dois solos, com dois sistemas de manejo e sem e com aplicação de $100 \mathrm{~kg} \mathrm{ha}^{-1}$ de $\mathrm{P}_{2} \mathrm{O}_{5}$ (superfosfato simples), sendo considerado um fatorial $2^{3}$, com um total de oito tratamentos dispostos em blocos ao acaso, com quatro repetiç̃es. Em todas as parcelas, foram depositados resíduos de soja para simular o que ocorreria na lavoura com cultivo contínuo. Esses resíduos foram cortados manualmente, com comprimento de aproximadamente $10 \mathrm{~cm}$, incluindo folhas e talos. A quantidade de resíduos depositada foi equivalente a 6,0 t ha-1 para simular condições de alta produtividade no campo. $\mathrm{O} \mathrm{P}$ foi aplicado superficialmente no momento da semeadura da soja, sendo depositado abaixo dos resíduos de soja.

A coleta da solução do solo foi realizada com instalação de uma cápsula porosa de porcelana na profundidade de 15 a $20 \mathrm{~cm}$, considerando que até $20 \mathrm{~cm}$ há maior exploração radicular por nutrientes pela cultura. Essa coleta foi realizada com o auxílio de uma bomba com pressão negativa de $46,662 \mathrm{kPa}$ (350 $\mathrm{mm} \mathrm{Hg})$, mantida durante $30 \mathrm{~min}$, tempo necessário para preenchimento das cápsulas de coleta. Posteriormente, com a troca da mangueira de sucção da bomba, as cápsulas foram esvaziadas e a solução coletada em tubos de vidro (10 a $15 \mathrm{~mL})$, sendo armazenadas em refrigerador $\left(4^{\circ} \mathrm{C}\right)$. A coleta de solução do solo iniciou-se na emergência e posteriormente entre 6 e 10 dias, até 55 dias após a emergência, considerando cada coleta como uma época. Esta solução do solo foi analisada em espectrômetro de emissão atômica em plasma induzido (ICP-AES), onde foram determinados os teores dos nutrientes $\mathrm{K}, \mathrm{Ca}$ e $\mathrm{Mg}$.

Após a colheita da parte aérea da soja, os vasos foram desmontados, coletando-se amostras de solo nas profundidades de $0-5,5-10,10-15,15-25$ e $25-40 \mathrm{~cm}$. Nessas amostras, foram determinados o $\mathrm{pH}$ e os teores de $\mathrm{K}^{+}$(Tedesco et al., 1995), matéria orgânica, $\mathrm{Ca}^{2+}$, $\mathrm{Mg}^{2+} \mathrm{e} \mathrm{Al}^{3+}$ (Raij et al., 2001). Estas análises foram realizadas no Departamento de Produção Vegetal, FCA-UNESP, Botucatu-SP. As análises estatísticas foram realizadas com o auxílio do programa SAS 8.2 (SAS, 2001) As comparações de médias foram realizadas utilizando-se o teste $\mathrm{F}$. 
Quadro 1. Caracterização química e granulométrica dos solos no campo, no momento da coleta das parcelas para constituição das unidades experimentais

\begin{tabular}{|c|c|c|c|c|c|}
\hline \multirow{2}{*}{ Solo e sistema de manejo } & \multicolumn{5}{|c|}{ Profundidade do solo (cm) } \\
\hline & $0-5$ & $5-10$ & $10-15$ & $15-25$ & $25-40$ \\
\hline & \multicolumn{5}{|c|}{$\mathrm{pH} \mathrm{CaCl}{ }_{2}$ do solo } \\
\hline LVA - SCC & 5,73 & 5,77 & 5,85 & 5,30 & 4,80 \\
\hline LVA - SSD & 5,95 & 4,92 & 4,45 & 4,34 & 4,53 \\
\hline LV - SCC & 5,03 & 5,22 & 5,24 & 4,42 & 4,28 \\
\hline \multirow[t]{2}{*}{ LV - SSD } & 5,60 & 4,37 & 3,97 & 4,07 & 4,16 \\
\hline & \multicolumn{5}{|c|}{ Matéria orgânica $\left(\mathrm{g} \mathrm{kg}^{-1}\right)$} \\
\hline LVA - SCC & 45,1 & 46,6 & 45,6 & 34,4 & 27,8 \\
\hline LVA - SSD & 53,9 & 44,2 & 42,3 & 38,7 & 27,8 \\
\hline LV - SCC & 48,7 & 47,5 & 49,2 & 41,3 & 33,0 \\
\hline \multirow[t]{2}{*}{ LV - SSD } & 55,4 & 50,6 & 46,1 & 35,2 & 26,6 \\
\hline & \multicolumn{5}{|c|}{ Fósforo $\left(\mathrm{mg} \mathrm{kg}^{-1}\right)$} \\
\hline LVA - SCC & 6,6 & 5,9 & 6,3 & 1,0 & 0,1 \\
\hline LVA - SSD & 12,2 & 36,7 & 11,2 & 1,8 & 0,1 \\
\hline LV - SCC & 8,7 & 10,8 & 10,0 & 3,2 & 0,6 \\
\hline \multirow[t]{2}{*}{ LV - SSD } & 10,8 & 20,0 & 13,6 & 5,3 & 0,3 \\
\hline & \multicolumn{5}{|c|}{ Potássio $\left(\mathrm{mmol}_{\mathrm{c}} \mathrm{kg}^{-1}\right)$} \\
\hline LVA - SCC & 5,1 & 6,9 & 5,7 & 3,5 & 1,0 \\
\hline LVA - SSD & 4,2 & 2,2 & 1,4 & 1,0 & 0,6 \\
\hline LV - SCC & 4,7 & 6,1 & 5,8 & 2,8 & 1,9 \\
\hline \multirow{2}{*}{ LV - SSD } & 6,4 & 5,3 & 3,2 & 2,3 & 1,9 \\
\hline & \multicolumn{5}{|c|}{ Cálcio $\left(\mathrm{mmol}_{\mathrm{c}} \mathrm{kg}^{-1}\right)$} \\
\hline LVA - SCC & 41,7 & 40,0 & 43,0 & 20,6 & 9,9 \\
\hline LVA - SSD & 59,3 & 28,3 & 13,9 & 8,2 & 7,7 \\
\hline LV - SCC & 46,2 & 49,4 & 50,7 & 17,1 & 8,4 \\
\hline \multirow[t]{2}{*}{ LV - SSD } & 46,9 & 34,3 & 13,2 & 7,0 & 6,2 \\
\hline & \multicolumn{5}{|c|}{ Magnésio $\left(\mathrm{mmol}_{\mathrm{c}} \mathrm{kg}^{-1}\right)$} \\
\hline LVA - SCC & 22,1 & 21,7 & 23,6 & 9,9 & 2,9 \\
\hline LVA - SSD & 32,0 & 7,0 & 3,6 & 1,9 & 1,9 \\
\hline LV - SCC & 20,7 & 22,9 & 21,4 & 4,8 & 1,4 \\
\hline \multirow[t]{2}{*}{ LV - SSD } & 18,8 & 7,2 & 1,4 & 0,5 & 0,5 \\
\hline & \multicolumn{5}{|c|}{ Alumínio $\left(\mathrm{mmol}_{\mathrm{c}} \mathrm{kg}^{-1}\right)$} \\
\hline LVA - SCC & 0,1 & 0,4 & 0,1 & 0,6 & 1,0 \\
\hline LVA - SSD & 0,3 & 1,5 & 5,1 & 5,9 & 1,4 \\
\hline LV - SCC & 0,4 & 0,4 & 0,4 & 7,4 & 13,8 \\
\hline \multirow[t]{2}{*}{ LV - SSD } & 1,0 & 7,4 & 17,4 & 15,3 & 10,5 \\
\hline & \multicolumn{5}{|c|}{ Argila $\left(\mathrm{g} \mathrm{kg}^{-1}\right)$} \\
\hline LVA - SCC & 500 & 520 & 483 & 592 & 696 \\
\hline LVA - SSD & 407 & 634 & 645 & 636 & 699 \\
\hline LV - SCC & 687 & 672 & 671 & 716 & 703 \\
\hline \multirow[t]{2}{*}{ LV - SSD } & 703 & 752 & 766 & 710 & 711 \\
\hline & \multicolumn{5}{|c|}{ Silte $\left(\mathrm{g} \mathrm{kg}^{-1}\right)$} \\
\hline LVA - SCC & 493 & 472 & 509 & 403 & 290 \\
\hline LVA - SSD & 582 & 363 & 350 & 358 & 297 \\
\hline LV - SCC & 238 & 248 & 256 & 208 & 217 \\
\hline \multirow[t]{2}{*}{ LV - SSD } & 205 & 164 & 152 & 210 & 220 \\
\hline & & & Areia ( $\mathrm{g} \mathrm{k}$ & & \\
\hline LVA - SCC & 7 & 8 & 8 & 5 & 14 \\
\hline LVA - SSD & 11 & 3 & 5 & 6 & 4 \\
\hline LV - SCC & 75 & 80 & 73 & 76 & 80 \\
\hline LV - SSD & 92 & 84 & 82 & 80 & 69 \\
\hline
\end{tabular}

\section{RESULTADOS E DISCUSSÃO}

De modo geral, as concentrações de K e Ca na solução do solo foram maiores nos primeiros dias de cultivo da soja, mantendo-se relativamente altas até 25 dias após a emergência, em ambos os solos e manejos adotados (Figura 1a,b). Após os 25 dias, houve uma drástica redução na concentração desses nutrientes em solução, o que pode ser explicado pelo aumento na demanda pela soja, devido ao crescimento intensivo da parte aérea e também pela maior área de exploração e absorção radicular. A aplicação de superfosfato simples influenciou na concentração tanto de $\mathrm{K}$ como de $\mathrm{Ca}$ e $\mathrm{Mg}$ na solução do solo, 
principalmente nas épocas 15 e 25 dias após a emergência da soja (Quadro 2). É importante ressaltar que o Ca também faz parte da composição do superfosfato simples, o que indiretamente pode ter afetado a concentração de $\mathrm{K}$ e $\mathrm{Mg}$ na solução, pelo aumento de sua concentração em solução e possível competição nos sítios de troca catiônica do solo.

Por ocasião da emergência da soja, o sistema de semeadura direta (SSD) mostrou menor concentração de K em solução com relação ao convencional (SCC) em ambos os solos (Figura 1a). Para o solo LV, a partir da segunda época de coleta, seis dias após a
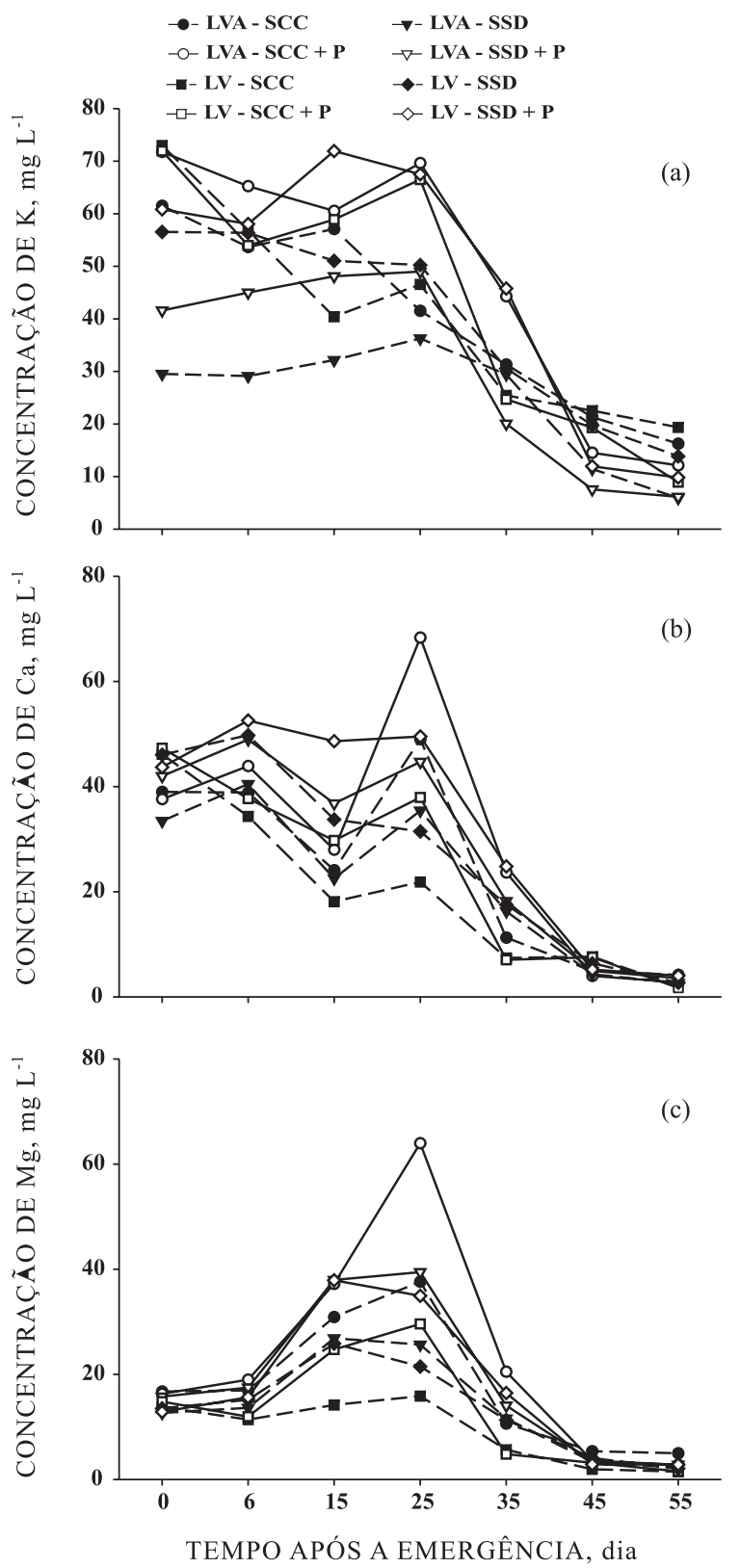

Figura 1. Concentrações de potássio (a), cálcio (b) e magnésio (c) na solução de dois Latossolos durante o ciclo de crescimento da soja, em função do manejo e da fertilização fosfatada.
Quadro 2. Significância das análises de variância para os teores de macronutrientes catiônicos da solução do solo

\begin{tabular}{lccc}
\hline \multirow{2}{*}{ Fonte de variação } & \multicolumn{3}{c}{ Cátions } \\
\cline { 2 - 4 } & K & Ca & Mg \\
\hline Solos & $*$ & $\mathrm{~ns}$ & $*$ \\
Manejo & $*$ & $*$ & $\mathrm{~ns}$ \\
Doses P & $*$ & $*$ & $*$ \\
Épocas & $*$ & $*$ & $*$ \\
Solos x Manejo & $*$ & $*$ & $*$ \\
Solos x Doses P & $\mathrm{ns}$ & $\mathrm{ns}$ & $\mathrm{ns}$ \\
Solos x Épocas & $\mathrm{ns}$ & $*$ & $*$ \\
Manejo x Doses P & $\mathrm{ns}$ & $\mathrm{ns}$ & $\mathrm{ns}$ \\
Manejo x Épocas & $*$ & $*$ & $*$ \\
Doses P x Épocas & $*$ & $*$ & $*$ \\
Solos x Manejo x Doses P & $\mathrm{ns}$ & $\mathrm{ns}$ & $\mathrm{ns}$ \\
Solos x Manejo x Épocas & $\mathrm{ns}$ & $*$ & $*$ \\
Solos x Doses P x Épocas & $\mathrm{ns}$ & $\mathrm{ns}$ & $\mathrm{ns}$ \\
Manejo x Doses P x Épocas & $\mathrm{ns}$ & $\mathrm{ns}$ & $\mathrm{ns}$ \\
Solos x Manejo x Doses P x Épocas & $\mathrm{ns}$ & $\mathrm{ns}$ & $\mathrm{ns}$
\end{tabular}

*: significativo a $5 \%$ pela análise da variância. ${ }^{\text {ns: }}$ não significativo.

emergência, a concentração de K foi semelhante para os dois sistemas de cultivo, o que pode ser resultante de maior poder de tamponamento por $\mathrm{K}$ deste solo, uma vez que ele tem teores maiores de MO e argila (Quadro 1). Para o solo LVA, a diferença se manteve até pelo menos 35 dias após a emergência. Isso mostra que o $\mathrm{K}$ pode ter sua disponibilidade em solução reduzida no sistema de semeadura direta, como possível complexação deste nutriente com compostos orgânicos ou grupos funcionais do solo.

A concentração de Ca em solução não apresentou variação significativa para o fator solo (Quadro 2), mas houve variação para manejo e adubação fosfatada. Pode-se destacar que, entre seis e 35 dias após a emergência, a concentração de Ca foi maior no SSD que no SCC para o solo LV. Já para o solo LVA, esta diferença não foi significativa (Figura 1b). Assim como visto para o $\mathrm{K}$, a concentração de Ca reduziu-se expressivamente após os 25 dias de emergência, como consequência da maior exigência pela cultura da soja.

A concentração de Mg na solução do solo foi maior nas avaliações realizadas aos 15 e 25 dias após a emergência da soja, em relação às demais épocas, sendo este efeito significativo para solo e para a aplicação de fertilizante fosfatado (Figura 1c e Quadro 2). No entanto, não foi observada diferença significativa entre sistemas de manejo para o Mg em solução quando se avalia todo o período de crescimento da soja. A aplicação de fertilizante fosfatado resultou em incremento na concentração de $\mathrm{Mg}$ em solução nas coletas realizadas desde 6 até 35 dias após a emergência, como resultado da competição promovida pela dissolução do fosfato, com o Ca ocupando os sítios de troca, deslocando $\mathrm{Mg}$ para a solução, como 
comentado anteriormente. É importante ressaltar aqui que houve efeito significativo para os cátions da solução do solo na interação solos x manejo, resultado que expressa as características de cada solo e a forma de manejo com que foi conduzido ao longo dos últimos anos, o que provavelmente vai se refletir também nas análises de solo apresentadas na sequência.

$\mathrm{O} \mathrm{pH}$ do solo no SSD foi maior que no convencional na camada mais superficial, sendo menor a partir da camada de 5-10 cm para ambos os solos (Figura 2a e Quadro 3), o que tem sido observado também por outros pesquisadores em áreas de produção e de pesquisa (Silveira et al., 2000; Martinazzo, 2006; Siqueira Neto, 2006). Maiores valores de $\mathrm{pH}$ observados no sistema convencional, a partir de 5$10 \mathrm{~cm}$ de profundidade, são ocasionados pelo revolvimento do solo, que promove uma homogeneização dos materiais no perfil do solo pelo menos até $15 \mathrm{~cm}$. Para o SSD, a deposição e decomposição de resíduos na superfície têm favorecido a manutenção do $\mathrm{pH} \mathrm{CaCl}_{2}$ entre 5,4 e 6,5, sendo considerado adequado para as plantas na camada mais superficial, uma vez que o $\mathrm{pH}$ dos resíduos normalmente se situa próximo de 6,0 até 6,5 (Pavinato, 2007). Em camadas mais profundas, esse efeito é inexpressivo e os processos naturais de acidificação promovem diminuição do $\mathrm{pH}$, o que poderá ser problema para o desenvolvimento de plantas, especialmente se houver $\mathrm{Al}^{3+}$ em concentração tóxica às plantas, como mostra a figura $2 \mathrm{~b}$. Os dois solos mostraram comportamento semelhante do $\mathrm{pH}$ ao longo do perfil do solo, diferindo estatisticamente entre os sistemas de manejo e os solos.

Os teores de $\mathrm{Al}$ extraídos por $\mathrm{KCl}$ 1,0 $\mathrm{mol} \mathrm{L}^{-1}$ são inversamente relacionados com os valores de $\mathrm{pH}$ do solo, pois, com a elevação do $\mathrm{pH}$, diminui a acidez trocável do solo, e vice-versa. Essa acidez trocável é composta por $\mathrm{H}$ e $\mathrm{Al}$ trocáveis, os quais se dissociam facilmente dos complexos de troca do solo (Sousa et al., 2007). Nos dados apresentados aqui, pode-se constatar que a diminuição do $\mathrm{pH}$ em profundidade no SSD foi diretamente relacionada com o aumento do teor de Al trocável (Figura $2 \mathrm{~b}$ e Quadro 3). Esse resultado é normalmente obtido no SSD, devido ao não revolvimento do solo e à deposição de resíduos de plantas, com acumulação de bases proveniente dos resíduos nas camadas mais superficiais. O SSD apresentou teor de $\mathrm{Al}$ trocável baixo na camada de 0 $5 \mathrm{~cm}$, semelhante ao sistema convencional, sendo significativamente superior ao convencional em camadas mais profundas, principalmente para o LV (Quadro 1). Quanto aos solos, o LV tem mais $\mathrm{Al}$ trocável que o LVA, principalmente no manejo SSD, mas essa característica foi significativa somente para camadas abaixo de $5 \mathrm{~cm}$. A explicação do baixo teor de Al trocável na camada $0-5 \mathrm{~cm}$ está nos altos valores de $\mathrm{pH}$ e de cátions trocáveis nesta camada, especialmente $\mathrm{Ca}^{2+}$ e $\mathrm{Mg}^{2+}$, uma vez que, com $\mathrm{pH}$ $\mathrm{CaCl}_{2}$ superior a 4,9 (Figura 2a), praticamente todo o $\mathrm{Al}$ do solo está precipitado como $\mathrm{Al}(\mathrm{OH})_{3}$. Neste caso,

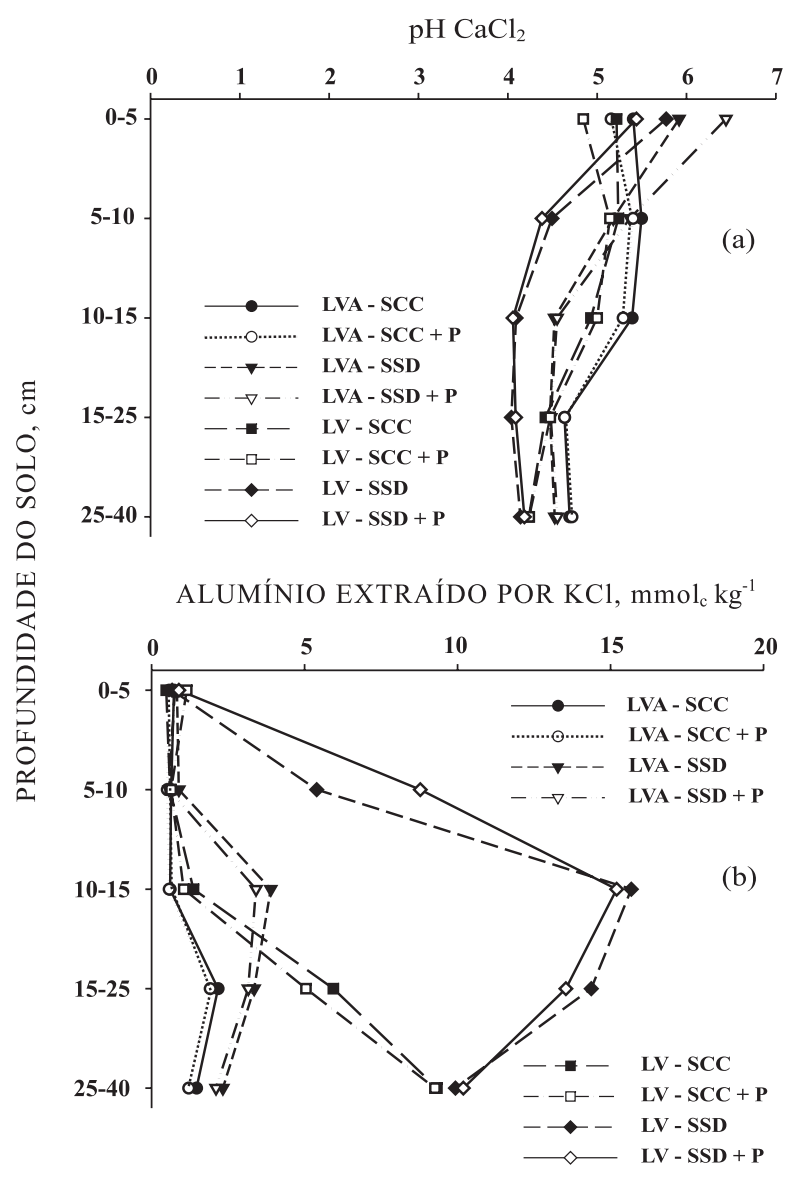

Figura 2. Valores de $\mathrm{pH} \mathrm{CaCl}_{2}$ (a) e teores de acidez trocável (b) nos solos LVA e LV sob dois sistemas de manejo (SCC e SSD), sem e com fertilizante fosfatado $(+\mathrm{P})$.

grande parte da CTC é ocupada por cátions importantes na fertilidade do solo, como o $\mathrm{Ca}$ e $\mathrm{Mg}$, como mencionado anteriormente.

De acordo com dados apresentados por vários autores, o SSD e cultivo mínimo, quando associados à rotação de culturas anuais, aumentam os teores de matéria orgânica (Paiva et al., 1997), a capacidade de troca catiônica (Souza \& Alves, 2003), bem como as bases trocáveis e o P disponível (Selles et al., 1997; Franchini et al., 1999; Amaral et al., 2004), além de haver diminuição do teor de $\mathrm{Al}^{3+}$ (Silveira \& Stone, 2001; Ciota et al., 2002; Almeida et al., 2005). Neste trabalho, foram observadas menores concentrações de Al trocável no SSD na camada superficial $(0-5 \mathrm{~cm})$ para o solo $L V$, e nas camadas $0-5$ e $5-10 \mathrm{~cm}$ para o LVA, como possível efeito da deposição dos resíduos e complexação do $\mathrm{Al}$ com compostos orgânicos do solo. No sistema convencional, os valores de $\mathrm{Al}$ trocável foram baixos em toda a camada arável, pelo menos até $15 \mathrm{~cm}$ (Figura $2 \mathrm{~b}$ ).

Houve aumento nos teores de matéria orgânica do solo na camada superficial $(0-5 \mathrm{~cm})$ ao longo dos anos 
Quadro 3. Significâncias das análises de variância para as características químicas do solo avaliadas em diferentes profundidades

\begin{tabular}{|c|c|c|c|c|c|c|}
\hline \multirow{2}{*}{ Fontes de variação } & \multicolumn{6}{|c|}{ Característica química do solo } \\
\hline & $\mathrm{pH} \mathrm{CaCl}{ }_{2}$ & $\mathrm{Al}^{3+}$ & MO & $\mathbf{K}$ & $\mathrm{Ca}^{2+}$ & $\mathrm{Mg}^{2+}$ \\
\hline \multicolumn{7}{|c|}{ Profundidade de $0-5 \mathrm{~cm}$} \\
\hline Solos & * & $\mathrm{ns}$ & $*$ & $*$ & * & $\mathrm{ns}$ \\
\hline Manejo & * & $\mathrm{ns}$ & * & $\mathrm{ns}$ & * & * \\
\hline Doses P & $\mathrm{ns}$ & * & $\mathrm{ns}$ & * & ns & ns \\
\hline Solos x Manejo & $\mathrm{ns}$ & ns & $\mathrm{ns}$ & $\mathrm{ns}$ & $\mathrm{ns}$ & ns \\
\hline Solos x Doses P & * & * & $\mathrm{ns}$ & * & ns & * \\
\hline Manejo x Doses P & * & ns & $\mathrm{ns}$ & * & ns & * \\
\hline Solos x Manejo x Doses P & $\mathrm{ns}$ & ns & $\mathrm{ns}$ & $\mathrm{ns}$ & $\mathrm{ns}$ & $\mathrm{ns}$ \\
\hline \multicolumn{7}{|c|}{ Profundidade de $5-10 \mathrm{~cm}$} \\
\hline Solos & * & * & $*$ & $*$ & * & $\mathrm{ns}$ \\
\hline Manejo & * & * & ns & * & * & $*$ \\
\hline Doses P & $\mathrm{ns}$ & ns & ns & * & ns & ns \\
\hline Solos x Manejo & $*$ & $*$ & $\mathrm{~ns}$ & * & $*$ & $*$ \\
\hline Solos x Doses P & $\mathrm{ns}$ & ns & $\mathrm{ns}$ & $\mathrm{ns}$ & * & * \\
\hline Manejo x Doses P & $\mathrm{ns}$ & ns & $\mathrm{ns}$ & $\mathrm{ns}$ & $\mathrm{ns}$ & $\mathrm{ns}$ \\
\hline Solos x Manejo x Doses P & $\mathrm{ns}$ & ns & $\mathrm{ns}$ & $\mathrm{ns}$ & * & $*$ \\
\hline \multicolumn{7}{|c|}{ Profundidade de $10-15 \mathrm{~cm}$} \\
\hline Solos & * & * & * & $*$ & * & $\mathrm{ns}$ \\
\hline Manejo & * & * & * & * & * & * \\
\hline Doses $\mathrm{P}$ & $\mathrm{ns}$ & ns & $\mathrm{ns}$ & * & $\mathrm{ns}$ & $\mathrm{ns}$ \\
\hline Solos x Manejo & $\mathrm{ns}$ & $*$ & $\mathrm{~ns}$ & * & $*$ & $\mathrm{~ns}$ \\
\hline Solos x Doses P & $\mathrm{ns}$ & ns & $\mathrm{ns}$ & $\mathrm{ns}$ & $\mathrm{ns}$ & $\mathrm{ns}$ \\
\hline Manejo x Doses P & $\mathrm{ns}$ & $\mathrm{ns}$ & $\mathrm{ns}$ & $\mathrm{ns}$ & ns & $\mathrm{ns}$ \\
\hline Solos x Manejo x Doses P & $\mathrm{ns}$ & nd & $\mathrm{ns}$ & $\mathrm{ns}$ & ns & $\mathrm{ns}$ \\
\hline \multicolumn{7}{|c|}{ Profundidade de $15-25 \mathrm{~cm}$} \\
\hline Solos & * & * & $*$ & $*$ & * & * \\
\hline Manejo & * & * & $\mathrm{ns}$ & * & * & * \\
\hline Doses P & $\mathrm{ns}$ & $\mathrm{ns}$ & $\mathrm{ns}$ & $\mathrm{ns}$ & $\mathrm{ns}$ & ns \\
\hline Solos x Manejo & $*$ & $*$ & $*$ & $\mathrm{~ns}$ & $*$ & $*$ \\
\hline Solos x Doses P & $\mathrm{ns}$ & ns & $\mathrm{ns}$ & $\mathrm{ns}$ & $\mathrm{ns}$ & $\mathrm{ns}$ \\
\hline Manejo x Doses P & $\mathrm{ns}$ & $\mathrm{ns}$ & $\mathrm{ns}$ & $\mathrm{ns}$ & $\mathrm{ns}$ & $\mathrm{ns}$ \\
\hline Solos x Manejo x Doses P & $\mathrm{ns}$ & $\mathrm{ns}$ & $*$ & $\mathrm{~ns}$ & $\mathrm{~ns}$ & $\mathrm{~ns}$ \\
\hline \multicolumn{7}{|c|}{ Profundidade de $25-40 \mathrm{~cm}$} \\
\hline Solos & * & $*$ & * & $*$ & $*$ & $\mathrm{~ns}$ \\
\hline Manejo & * & $\mathrm{ns}$ & $\mathrm{ns}$ & * & $\mathrm{ns}$ & $\mathrm{ns}$ \\
\hline Doses P & $\mathrm{ns}$ & ns & $\mathrm{ns}$ & $\mathrm{ns}$ & $\mathrm{ns}$ & $\mathrm{ns}$ \\
\hline Solos x Manejo & $\mathrm{ns}$ & $\mathrm{ns}$ & $*$ & $\mathrm{~ns}$ & $*$ & $\mathrm{~ns}$ \\
\hline Solos x Doses P & $\mathrm{ns}$ & ns & * & $\mathrm{ns}$ & ns & $\mathrm{ns}$ \\
\hline Manejo x Doses P & $\mathrm{ns}$ & $\mathrm{ns}$ & $\mathrm{ns}$ & $\mathrm{ns}$ & $\mathrm{ns}$ & $\mathrm{ns}$ \\
\hline Solos x Manejo x Doses P & ns & ns & $\mathrm{ns}$ & $\mathrm{ns}$ & $\mathrm{ns}$ & $\mathrm{ns}$ \\
\hline
\end{tabular}

*: significativo a $5 \%$ pela análise da variância. ${ }^{\text {ns: }}$ não significativo.

de cultivo no SSD, quando comparado com o cultivo convencional (Figura 3a) para ambos os solos, tendo em vista que há deposição de resíduos das culturas sobre a superfície do solo no SSD, o que resulta em acumulação superficial de materiais orgânicos em diferentes estágios de decomposição. O teor de MO apresentou comportamento semelhante no perfil para os manejos dentro de cada solo, no entanto, o solo LV apresenta maior teor de MO em todas as camadas analisadas.

A semeadura direta, comparada ao sistema convencional, diminui a velocidade de degradação da matéria orgânica (Fontana et al., 2006), pelo menor contato entre o resíduo vegetal e o solo, por ser depositado sobre a superfície, retardando o ataque pelos microrganismos do solo. Hermani et al. (1999) e Almeida et al. (2005) observaram que no SSD houve aumento do estoque de matéria orgânica nas camadas superficiais do solo, e, consequentemente, aumento nos teores de nutrientes nas frações orgânicas, resultando em maior estabilidade produtiva do sistema de semeadura direta em comparação ao convencional.

Quanto ao K disponível no solo, os teores na camada superficial $(0-5 \mathrm{~cm})$ não foram alterados com os manejos do solo. Somente houve variação entre os solos avaliados e com a dose de fertilizante fosfatado (Figura 3b). As demais profundidades, houve efeito de solo e manejo (Quadro 3). No solo LV, o cultivo 


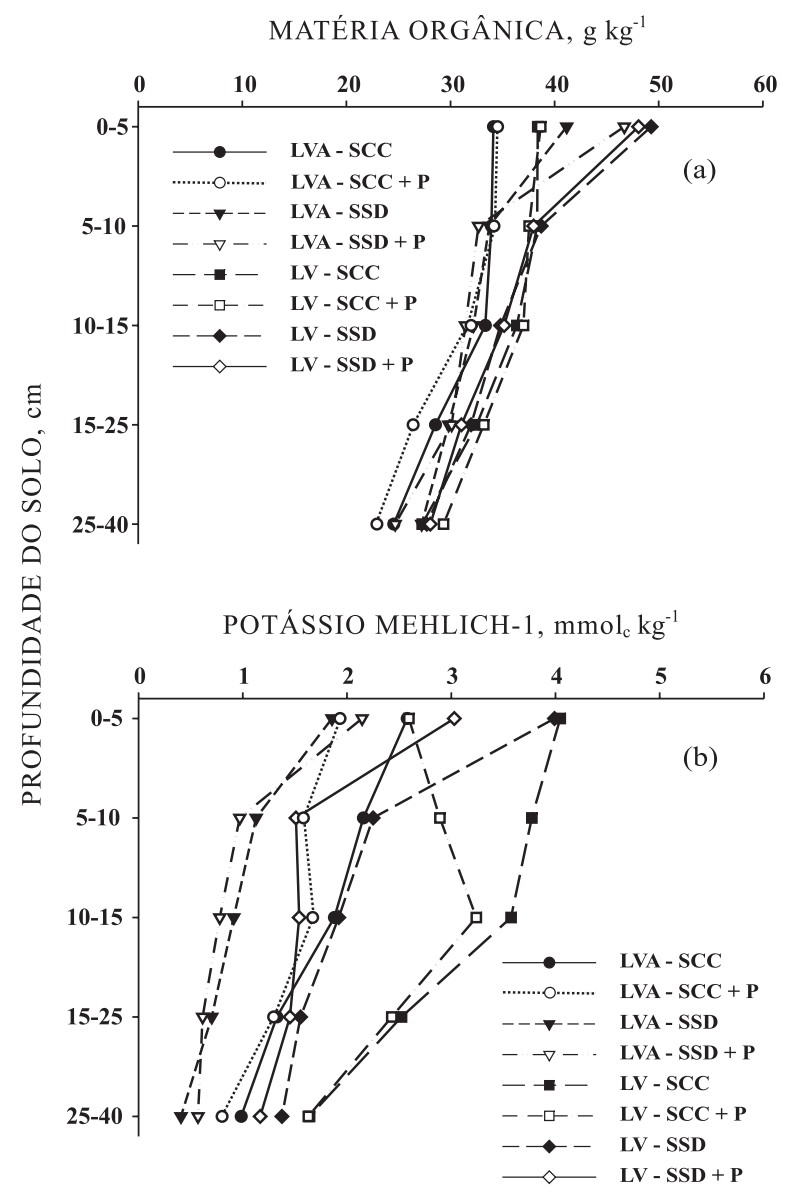

Figura 3. Teores de matéria orgânica (a) e de potássio (b) disponível nos solos LVA e LV sob dois sistemas de manejo (SCC e SSD), com e sem fertilizante fosfatado $(+\mathrm{P})$.

convencional teve alto teor de $\mathrm{K}$ disponível até a profundidade revolvida pelo sistema de manejo (pelo menos $15 \mathrm{~cm}$ ), com redução gradual após esta profundidade. Já para o SSD, os teores foram altos em superfície, com redução gradual após a camada superficial. Essa mesma tendência foi obtida tanto para o SCC como para o SSD no solo LVA. Esse comportamento do K disponível na camada superficial em função do manejo é resultante da deposição de resíduos superficialmente no SSD, sendo disponibilizada maior quantidade deste nutriente nesta camada pela decomposição, em detrimento de camadas mais profundas. Já no SCC, como esses resíduos foram incorporados, houve liberação de K uniformemente em toda a camada arável. A possível perda de K no perfil do solo com o SSD também é uma hipótese não descartada, pois foi observado menor teor de K disponível com esse manejo em todas as camadas avaliadas do perfil.

Na média dos solos, o LVA apresentou teor médio de K disponível inferior ao solo LV. Quanto aos manejos, o SCC apresentou maior teor de K disponível que o SSD (Quadro 3). Outro ponto a ser ressaltado é que houve efeito significativo da fertilização fosfatada na disponibilidade de $\mathrm{K}$ no solo até $10-15 \mathrm{~cm}$, possivelmente havendo reação do fosfato com o K, reduzindo a concentração de K na solução do solo, especialmente nas camadas mais superficiais. Em trabalho desenvolvido para adaptação de amostragens de solo ao plantio direto, Silveira et al. (2000) observaram que, na profundidade de $0-5 \mathrm{~cm}$, o maior teor de $\mathrm{K}$ disponível ocorreu no SSD, quando comparado com cultivo convencional. No entanto, esses autores observaram menor teor de $\mathrm{K}$ disponível na camada de 5-20 cm, o que mostra que este cátion concentrou-se na camada superficial do solo. Outros autores também têm observado maior teor de $\mathrm{K}$ na camada mais superficial no SSD em relação ao SCC (Oliveira \& Pavan, 1996; Almeida et al., 2005), embora em alguns solos não seja observada diferença entre os manejos quanto ao teor de $\mathrm{K}$ disponível no perfil até $30 \mathrm{~cm}$ de profundidade (Franchini et al., 2000).

Quanto ao teor de Ca trocável no solo, os dados têm demonstrado que realmente há acumulação deste nutriente em camadas superficiais do solo no SSD, o que muitas vezes pode representar redução de disponibilidade em camadas mais profundas, comparado com o SCC (Figura 4a). Em ambos os solos foram constatados teores altos de Ca disponível na camada de 0-5 cm para o SSD, com redução drástica já na segunda camada $(5-10 \mathrm{~cm})$. No sistema convencional, os teores de Ca trocável foram semelhantes no perfil, desde a superfície até $15 \mathrm{~cm}$, em ambos os solos, profundidade esta que é revolvida no momento do preparo do solo. Diferenças significativas foram observadas no teor de Ca trocável tanto para o fator solo como para o fator manejo, em praticamente todas as camadas, inclusive com interação entre estes dois fatores em algumas camadas (Quadro 3). Almeida et al. (2005) observaram uma tendência de aumento no teor de Ca trocável no SSD com rotação de culturas, em relação ao sistema convencional, até $30 \mathrm{~cm}$ de profundidade, em um Cambissolo de Santa Catarina, não sendo constatada acumulação de Ca na superfície do SSD.

O Mg trocável no solo apresentou grande concentração na camada superficial no SSD e redução drástica nas camadas mais profundas (Figura $4 \mathrm{~b}$ ). Também no sistema convencional os teores se mantiveram altos até $10-15 \mathrm{~cm}$, semelhante ao comportamento observado para o teor de Ca trocável, mas em escala inferior. O sistema de manejo modificou significativamente o teor de $\mathrm{Mg}$ trocável do solo (Quadro 3), com acúmulo expressivo no SSD, na camada de $0-5 \mathrm{~cm}$, em detrimento das camadas mais profundas, onde o SCC apresentou maiores valores. Estes resultados estão de acordo com os apresentados por Silveira et al. (2000), que também constataram maior concentração dos cátions $\mathrm{Ca}$ e $\mathrm{Mg}$ na camada superficial do solo $(0-5 \mathrm{~cm})$ no SSD, devido à sorção desses dois elementos pelas cargas negativas da superfície do solo, facilitando sua permanência nesta camada. 


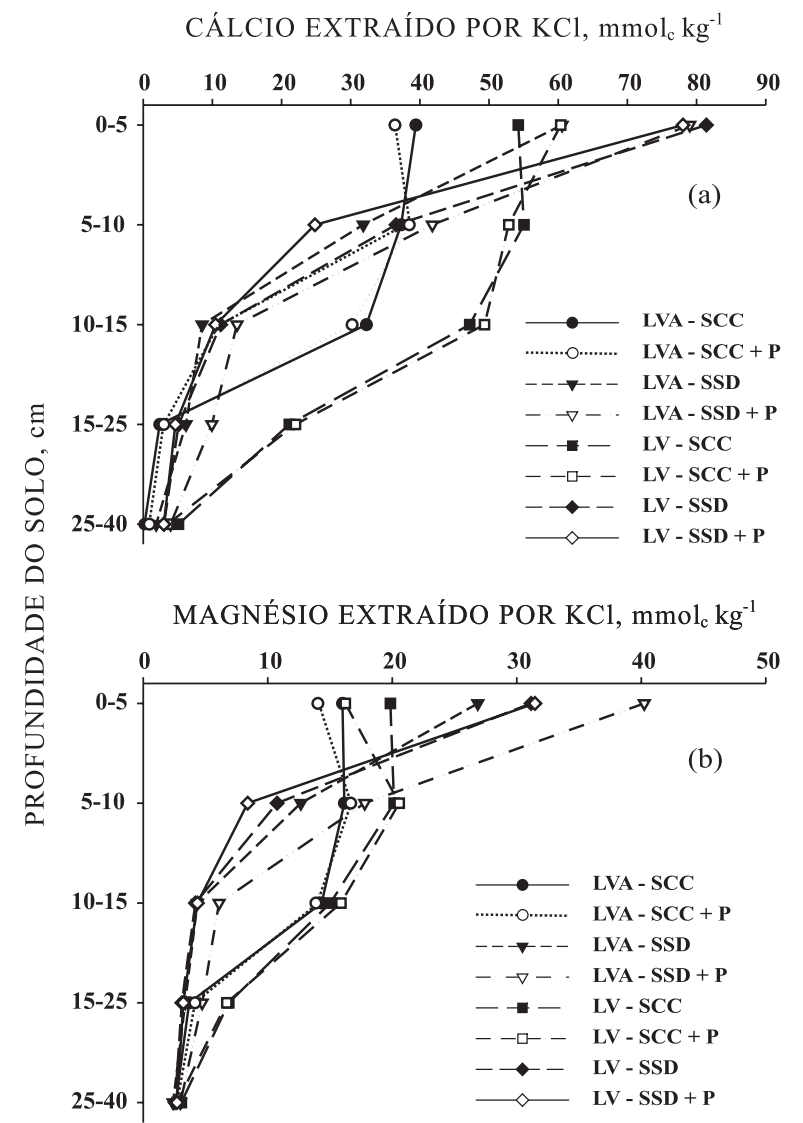

Figura 4. Teores de cálcio (a) e de magnésio trocável (b) nos solos LVA e LV sob dois sistemas de manejo (SCC e SSD), com e sem fertilizante fosfatado $(+\mathrm{P})$.

De modo geral, foram observadas alterações expressivas na acidez e nos teores de cátions do solo com a adoção do SSD. Houve elevação da disponibilidade de $\mathrm{Ca}$ e $\mathrm{Mg}$ na camada mais superficial do solo $(0-5 \mathrm{~cm})$, em detrimento de camadas mais profundas, cujos valores foram inferiores ao SCC. Já para o $\mathrm{K}$, houve redução da disponibilidade com a adoção do SSD em relação ao SCC. Porém, o pH não foi alterado na camada mais superficial, mas a partir de $5 \mathrm{~cm}$ de profundidade houve redução do $\mathrm{pH}$ após alguns anos de adoção do sistema, quando comparado com a camada arável do SCC.

\section{CONCLUSÕES}

1. O sistema de cultivo promove mudanças na disponibilidade de $\mathrm{K}$ na camada considerada arável do perfil do solo (até $25 \mathrm{~cm}$ ), sendo observada menor disponibilidade no sistema de semeadura direta em relação ao sistema convencional. Solos com camadas superficiais mais argilosas, caso do LV, tem potencial de manter maior teor de $\mathrm{K}$ disponível nestas camadas.
2. Nos solos estudados, a disponibilidade de Ca e $\mathrm{Mg}$ é maior na camada superficial $(0-5 \mathrm{~cm})$ no sistema plantio direto, em consequência da disponibilização destes nutrientes dos resíduos em decomposição. Para o sistema convencional, a disponibilidade é menor na superfície, mas ainda em teores considerados altos, e mantém esta disponibilidade em toda a camada arável do perfil.

\section{AGRADECIMENTOS}

À Fapesp, pela concessão de bolsa e recursos para execução da referida pesquisa. Especial agradecimento à Empresa SLC Agrícola, pela concessão da área experimental para coleta dos solos, com a geração e publicação dos atuais dados.

\section{LITERATURA CITADA}

ALMEIDA, J.M.; BERTOL, I.; LEITE, D.; AMARAL, A.J. \& ZOLDAN Jr., W.A. Propriedades químicas de um cambissolo húmico sob preparo convencional e semeadura direta após seis anos de cultivo. R. Bras. Ci. Solo, 29:437445,2005 .

AMARAL, A.S.; ANGHINONI, I. \& DESCHAMPS, F.C. Resíduos de plantas de cobertura e mobilidade dos produtos da dissolução do calcário aplicado na superfície do solo. R. Bras. Ci. Solo, 28:115-123, 2004.

BAYER, C. \& MIELNICZUK, J. Características químicas do solo afetadas por métodos de preparo e sistemas de cultura. R. Bras. Ci. Solo, 21:105-112, 1997.

CIOTTA, M.N.; BAYER, C.; ERNANI, P.R.; FONTOURA, S.M.V.; ALBUQUERQUE, J.A. \& WOBETO, C. Acidificação de um Latossolo sob plantio direto. R. Bras. Ci. Solo, 26:1055-1064, 2002.

EMPRESA BRASILEIRA DE PESQUISA AGROPECUÁRIA EMBRAPA. Centro Nacional de Pesquisa do Solo. Sistema brasileiro de classificação de solos. Rio de Janeiro, 1999. $412 \mathrm{p}$.

EMPRESA BRASILEIRA DE PESQUISA AGROPECUÁRIA EMBRAPA. Serviço Nacional de Levantamento e Conservação de Solo. Manual de métodos de análise de solo. 2.ed. Rio de Janeiro, 1997. 212p.

FONTANA, A.; PEREIRA, M.G.; LOSS, A.; CUNHA, T.J.F. \& SALTON, J.C. Atributos de fertilidade e frações húmicas de um Latossolo Vermelho no Cerrado. Pesq. Agropec. Bras., 41:847-853, 2006.

FRANCHINI, J.C.; HOFFMANN-CAMPO, C.B.; TORRES, E.; MIYAZAWA, M. \& PAVAN, A. Organic composition of green manure during growth and its effect on cation mobilization in an acid Oxisol. Comm. Soil Sci. Plant Anal., 34:2045-2058, 2003. 
FRANCHINI, J.C.; BORKERT, C.M.; FERREIRA, M.M. \& GAUDÊNCIO, C.A. Alterações na fertilidade do solo em sistemas de rotação de culturas em semeadura direta. $R$. Bras. Ci. Solo, 24:459-467, 2000

FRANCHINI, J.C.; MIYAZAWA, M.; PAVAN, M.A. \& MALAVOLTA, E. Dinâmica de íons em solo ácido lixiviado com extratos de resíduos de adubos verdes e soluções puras de ácidos orgânicos. Pesq. Agropec. Bras., 34:2267. 2276,1999

FRAZAO, L.A.; PICCOLO, M.C.; FEIGL, B.J.; CERRI, C.C. \& CERRI, C.E.P. Propriedades químicas de um Neossolo Quartzarênico sob diferentes sistemas de manejo no Cerrado mato-grossense. Pesq. Agropec. Bras., 43:641$648,2008$.

HERMANI, L.C.; KURIHARA, C.H. \& SILVA, W.M. Sistemas de manejo de solo e perdas de nutrientes e matéria orgânica por erosão. R. Bras. Ci. Solo, 23:145-154, 1999.

KRETZSCHMAR, R.M.; HAFNER, H.; BATIONO, A. \& MARSCHNER, H. Long- and short-term effects of crop residues on aluminum toxicity, phosphorus availability and growth of pearl millet in an acid sandy soil. Plant Soil, 136:215-223, 1991.

MARTINAZZO, R. Diagnóstico da fertilidade de solos em áreas sob plantio direto consolidado. Santa Maria, Universidade Federal de Santa Maria, 2006. 82p. (Tese de Mestrado)

MIYASAWA, M.; PAVAN, M.A. \& CALEGARI, A. Efeito de material vegetal na acidez do solo. R. Bras. Ci. Solo, 17:411 416, 1993.

OLIVEIRA, E.L. \& PAVAN, M.A. Control of soil acidity in no tillage system for soybean production. Soil Till. Res., 38:4757, 1996.

PAIVA, P.L.R.; FURTINI NETO, A.E.; VALE, F.R. \& FAQUIN, V. Efeito do manejo do solo sobre os teores de matéria orgânica, nitrogênio mineral, fósforo e bases trocáveis. Ci. Agrotec., 21:35-43, 1997.

PAVINATO, P.S. Dinâmica do fósforo no solo em função do manejo e da presença de resíduos em superfície. Botucatu, Universidade Estadual Paulista, 2007. 145p. (Tese de Doutorado)
PAVINATO, P.S.; MERLIN, A. \& ROSOLEM, C.A. Organic compounds from plant extracts and their effect on soil phosphorus availability. Pesq. Agropec. Bras., 43:13791388, 2008.

RAIJ, B.van; ANDRADE, J.C.; CANTARELLA, H. \& QUAGGIO, J.A. Análise química de para avaliação da fertilidade de solos tropicais. Campinas, Instituto Agronômico, 2001. 285p.

SAS Institute. SAS user's guide: Statistics, Ver 8.2; Cary, 2001.

SELLES, F.; KOCHANN, R.A.; DENARDIN, J.E.; ZENTNER, R.P. \& FAGANELLO, A. Distribution of phosphorus fractions in a Brazilian oxisol under different tillage systems. Soil Till. Res., 44:23-34, 1997.

SILVEIRA, P.M. \& STONE, L.F. Teores de nutrientes e matéria orgânica afetados pela rotação de culturas e sistema de preparo do solo. R. Bras. Ci. Solo, 25:387-394, 2001.

SILVEIRA, P.M.; ZIMMERMANN, F.J.P.; SILVA, S.C. \& CUNHA, A.A. Amostragem e variabilidade espacial de características químicas de um Latossolo submetido a diferentes sistemas de preparo. Pesq. Agropec. Bras., 35:2057-2064, 2000.

SIQUEIRA NETO, M. Estoque de carbono e nitrogênio do solo com diferentes usos no cerrado em Rio Verde. Piracicaba, Universidade de São Paulo, 2006. 159p. (Tese de Doutorado)

SOUSA, D.M.G.; MIRANDA, L.N. \& OLIVEIRA, S.A. Acidez do solo e sua correção. In: NOVAIS, R.F.; ALVAREZ V., V.H.; BARROS, N.F.; FONTES, R.L.F.; CANTARUTTI, R.B. \& NEVES, J.C.L., eds. Fertilidade do solo. ViçosaMG, Sociedade Brasileira de Ciência do Solo, 2007. $1017 \mathrm{p}$.

SOUZA, Z.M. \& ALVES, M.C. Propriedades químicas de um Latossolo Vermelho distroférrico de cerrado sob diferentes usos e manejos. R. Bras. Ci. Solo, 27:133-139, 2003.

TEDESCO, M.J.; GIANELLO, C.; BISSANI, C.A.; BOHNEN, H. \& VOLKWEISS, S.J. Análises de solo, plantas e outros materiais. 2.ed. Porto Alegre, Universidade Federal do Rio Grande do Sul, 1995. 174p. (Boletim Técnico, 5) 